\title{
Interfacing medicinal chemistry with structural bioinformatics: implications for T box riboswitch RNA drug discovery
}

\author{
Franziska Jentzsch ${ }^{1,2}$, Jennifer $V$ Hines $^{2^{*}}$ \\ From Great Lakes Bioinformatics Conference 2011 \\ Athens, OH, USA. 2-4 May 2011
}

\begin{abstract}
Background: The T box riboswitch controls bacterial transcription by structurally responding to tRNA aminoacylation charging ratios. Knowledge of the thermodynamic stability difference between two competing structural elements within the riboswitch, the terminator and the antiterminator, is critical for effective $T$ boxtargeted drug discovery.

Methods: The $\triangle \mathrm{G}$ of aminoacyl tRNA synthetase (aaRS) $T$ box riboswitch terminators and antiterminators was predicted using DINAMelt and the resulting $\triangle \Delta G\left(\Delta G_{\text {Terminator }}-\Delta G_{\text {Antiterminator }}\right)$ values were compared.

Results: Average $\Delta \Delta G$ values did not differ significantly between the bacterial species analyzed, but there were significant differences based on the type of aaRS.

Conclusions: The data indicate that, of the bacteria studied, there is little potential for drug targeting based on overall bacteria-specific thermodynamic differences of the $T$ box antiterminator vs. terminator stability, but that aaRS-specific thermodynamic differences could possibly be exploited for designing drug specificity.
\end{abstract}

\section{Background}

The $\mathrm{T}$ box riboswitch is an important regulatory mechanism found in Gram-positive bacteria including many pathogens [1-3]. The riboswitch responds to high levels of uncharged (non-aminoacylated) tRNA to control the transcription of cognate genes (e.g., aminoacyl tRNA synthetase, aaRS, genes) [3]. Cognate, uncharged tRNA binds the 5'-untranslated region of $\mathrm{T}$ box mRNA during transcription and, when present in sufficient quantities, results in antitermination (Figure 1a) [3]. The tRNA anticodon binds a specifier sequence in Stem 1, thus providing the cognate specificity, while the uncharged tRNA acceptor end nucleotides bind the first four bases in a seven nucleotide bulge of the highly conserved antiterminator structural element [4]. Aminoacylated-tRNA also binds the specifier sequence, but does not bind the antiterminator element [5]. In the absence of uncharged

\footnotetext{
*Correspondence: hinesj@ohio.edu

2Department of Chemistry \& Biochemistry, Ohio University, Athens, OH, USA
} Full list of author information is available at the end of the article
tRNA bound to the antiterminator element, a more thermodynamically stable stem-loop structure forms (the terminator) followed by factor-independent transcription termination a few nucleotides later [3]. The antiterminator and the terminator are mutually exclusive structural elements due to sharing common nucleotides (Figure 1b). Ligands that target and disrupt the $\mathrm{T}$ box riboswitch function could be potential antibacterial agents in light of the critical genes regulated by the $\mathrm{T}$ box mechanism $[1,2]$.

We have been investigating the structure-function relationship of the $\mathrm{T}$ box antiterminator element and the key recognition features necessary for ligands to specifically bind the antiterminator and disrupt its function. There are few detailed medicinal chemistry studies of ligands targeting RNA [6] and this project has required an extensive multidisciplinary approach. The solution structure of antiterminator model RNA AM1A [7] was determined using molecular modelling with NMRderived constraints [8]. The structure indicated that the

\section{()




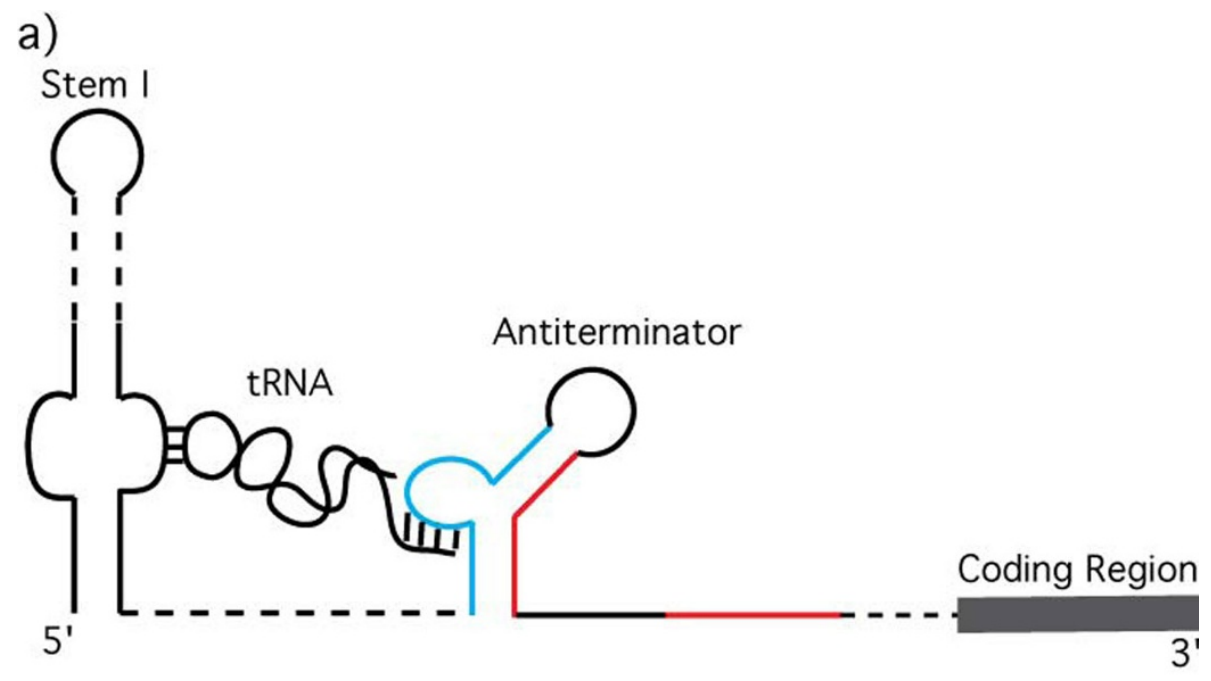

b)

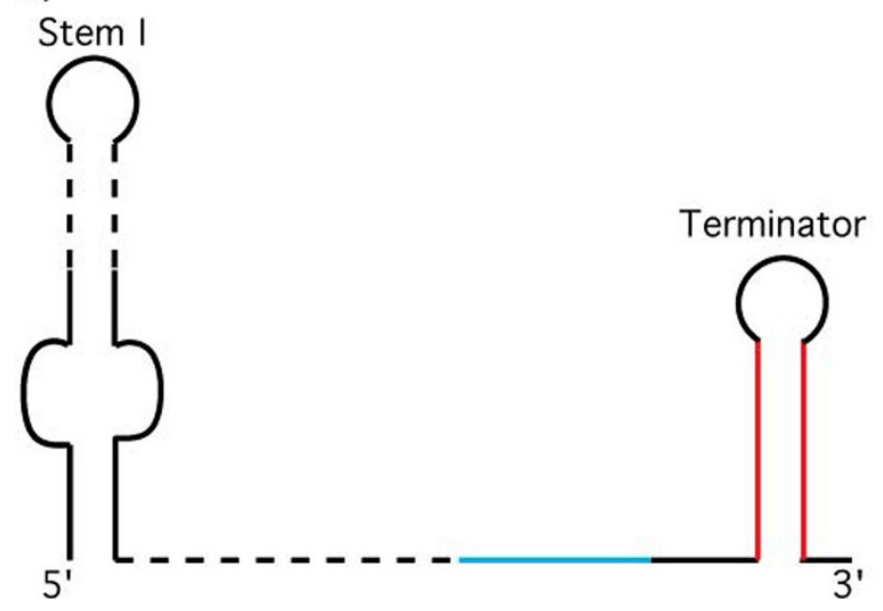

Figure $1 \mathrm{~T}$ box transcription antitermination mechanism. a) Uncharged, cognate tRNA binds the $5^{\prime}$-untranslated region of the nascent mRNA and stabilizes the antiterminator. b) In the absence of uncharged, cognate tRNA, the more stable terminator forms and transcription is terminated before the translation start site.

seven-nucleotide bulge of the antiterminator was not pre-ordered for tRNA binding, but rather, that binding of the tRNA acceptor end must require a certain extent of tertiary-structure capture and/or an induced fit. Fluorescence life-time studies confirmed that modest antiterminator structural reorganization occurs upon tRNA binding in a magnesium-dependent manner [9]. While some RNAs have specific divalent metal ion binding sites, for the antiterminator RNA, the $\mathrm{Mg}^{2+}$ binds via a diffuse, outer-sphere interaction [10]. In vitro selection studies of both the antiterminator [11] and tRNA [12] indicate that there are likely no direct interactions between the tRNA and the antiterminator other than the known base pairing between the acceptor end nucleotides and the first four nucleotides at the 5 -end of the seven-nucleotide bulge. Given this antiterminator structure-function information, ligands could potentially disrupt tRNA binding simply by competing with the base pairing between the tRNA acceptor end and the antiterminator bulge nucleotides.

Aminoglycosides bind AM1A in a structure-specific manner, most likely via electrostatics $[13,14]$. In contrast, two novel classes of heterocyclic compounds, triazoles and oxazolidinones, bind AM1A with structure-specificity and high affinity, but without reliance on electrostatics [15-18] and can alter $\mathrm{T}$ box transcription antitermination [16]. A computational, quantitative structure activity relationship analysis has shown that hydrophobic interactions play a significant role in the binding of these compounds to AM1A [19]. Furthermore, the small molecule ligands disrupt tRNA binding to the antiterminator in a structure-specific manner [20]. 
From a drug discovery perspective, a key factor to determine is the range of ligand-induced stabilization that can be accommodated without overly stabilizing the antiterminator element and precluding terminator formation. The goal of our $\mathrm{T}$ box drug discovery project is to determine the key ligand features that lead to specific antiterminator binding, but that do not result in excessive stabilization of the antiterminator secondary structure. These ligands could then potentially compete with tRNA for binding to the antiterminator, but still allow terminator formation such that transcription of a $\mathrm{T}$ box gene critical for bacterial survival would be terminated (Figure 2$)$. The predicted thermodynamic stability $(\Delta \mathrm{G})$ of the terminator and antiterminator structural elements have been compared for the B. subtilis tyrS T box [4]. However, there has been no systematic comparison of predicted thermodynamic stability differences for a larger set of $\mathrm{T}$ box genes. Using a structural bioinformatics approach, we have analyzed the differences in predicted free energy $(\Delta \Delta G)$ between antiterminators and

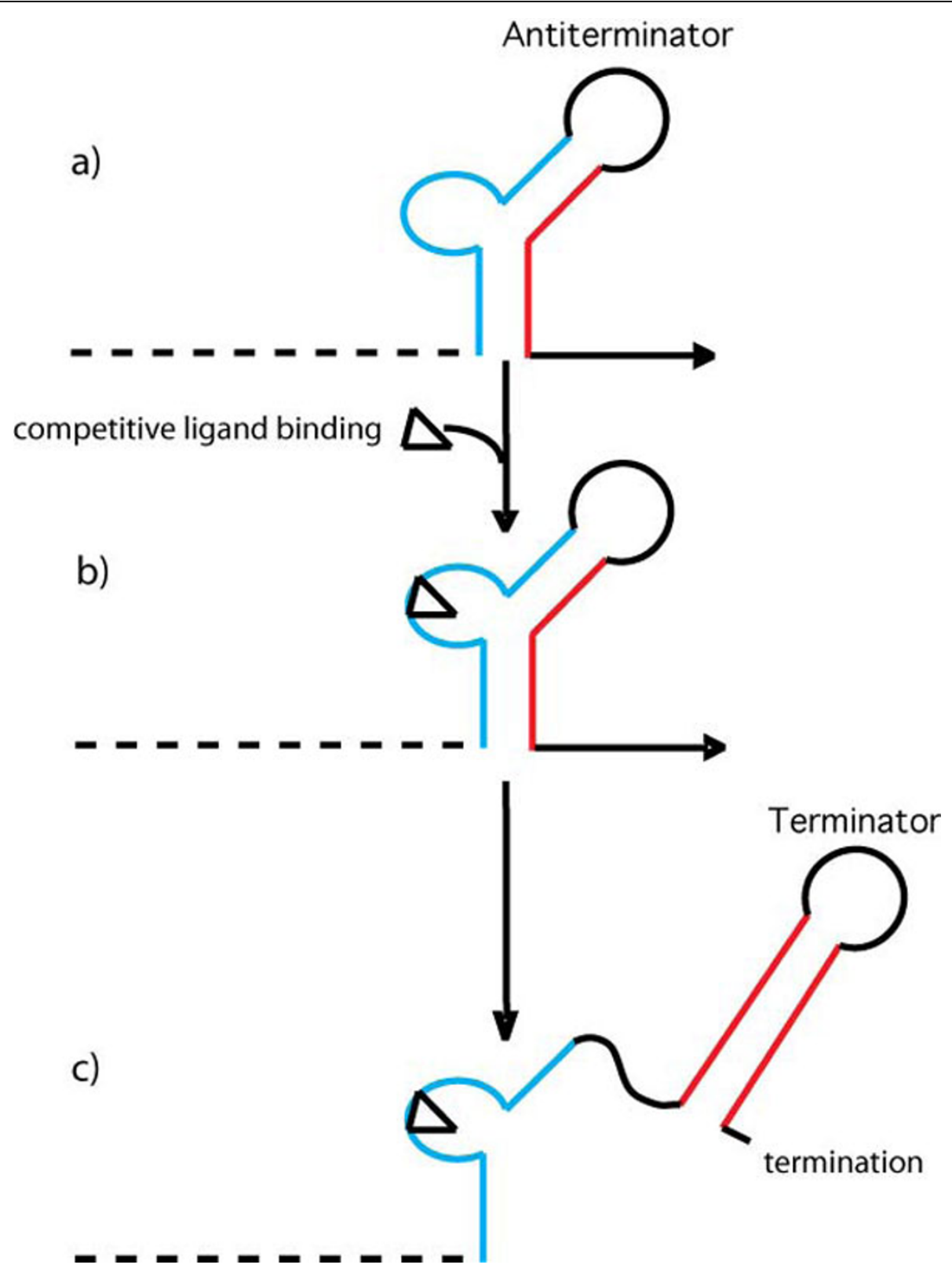

Figure $\mathbf{2}$ T box drug discovery goal. As soon as the antiterminator is transcribed (a), a small molecule ligand competes with tRNA for binding the antiterminator (b), but does not overly stabilize the antiterminator such that the terminator can form and transcription is terminated (c). 
terminators in a set of aaRS $\mathrm{T}$ box genes in order to predict an upper limit of ligand-induced stabilization that can potentially be accommodated.

\section{Methods}

The thermodynamic stability of $\mathrm{T}$ box antiterminator and terminator structural elements was calculated using the DINAMelt server [21]. The DINAMelt server computes the secondary structure and free energy of the folded RNA using a secondary structure folding algorithm and empirically-derived nearest neighbour coefficients [21]. The folding algorithm predicts the minimum energy RNA secondary structure using the available thermodynamic data for base pairing, base stacking and destabilizing energies $[22,23]$. The sequences analyzed were predicted to be involved in aaRS $\mathrm{T}$ box antiterminator and terminator structural elements from Bacillus cereus (BC), Bacillus subtilis (BS), Clostridium botulinum (CB), Clostridium difficile (CDF), Clostridium perfringens (CPE), Staphylococcus aureus Mu50 (SA), Streptococcus agalactiae (SAG), and, Streptococcus pyogenes (SPY) [2]. The $\Delta \mathrm{G}$ for each aaRS $\mathrm{T}$ box antiterminator and terminator sequence was determined using the Quickfold (RNA 3.0) option on the DINAMelt server and the predicted thermodynamic stability difference calculated from $\Delta \Delta G=\Delta G_{\text {Terminator }}$ $\Delta G_{\text {Antiterminator. }}$ The $\%$ suboptimal setting was adjusted as necessary to obtain the lowest free energy antiterminator fold that had the consensus secondary structure of the core seven-nucleotide bulge containing the 5' -UGGN-3' nucleotides that are complementary to the tRNA acceptor end nucleotides [4].

\section{Results and discussion}

The free energy values for the antiterminator and terminator structural elements of the $\mathrm{T}$ box genes analyzed were calculated using the DINAMelt webserver [21]. The predicted $\Delta G$ values are listed in Additional File 1 and the relative comparisons of terminator vs.antiterminator stability $(\Delta \Delta G)$ are summarized in Figure 3 . The overall average $\Delta \Delta G$ for all aaRS studied was $-12.8 \mathrm{kcal} /$ mol. The average $\Delta \Delta G$ values did not differ significantly between bacteria when comparing between the pathogenic bacteria nor between pathogenic vs. the nonpathogenic bacteria studied (BS) (Figure 3a). In contrast, there were significant differences in $\Delta \Delta G$ averages between specific aaRS with alanyl aaRS having the smallest average $\Delta \mathrm{G}(-7.8 \pm 3.5 \mathrm{kcal} / \mathrm{mol})$ and glycyl aaRS having the largest $(-20.1 \pm 4.6 \mathrm{kcal} / \mathrm{mol}$ ) (Figure $3 \mathrm{~b}$ ). Based on these results, the glycyl aaRS, on average, may be best able to accommodate ligand-induced stabilization of the antiterminator and still allow formation of the terminator. An important factor to consider, however, is that the free energy calculations are based on empirically-derived parameters for known RNA structural motifs [21]. Structural motifs, especially in loops and bulges, that have not been previously characterized might contribute to the stability of the RNA elements and not be accounted for in the DINAMelt $\Delta \mathrm{G}$ calculations. Since the loop of the antiterminator is not highly conserved [4], most likely there is no structural motif within it that might contribute to the antiterminator stability, however, the possibility cannot be excluded. Further investigation of experimentally-derived free energy values of individual antiterminators and terminators is needed.

\section{Conclusions}

The free energy of $\mathrm{T}$ box riboswitch antiterminator and terminator elements was predicted and compared for a series of aaRS $\mathrm{T}$ box genes. The observed aaRS-specific

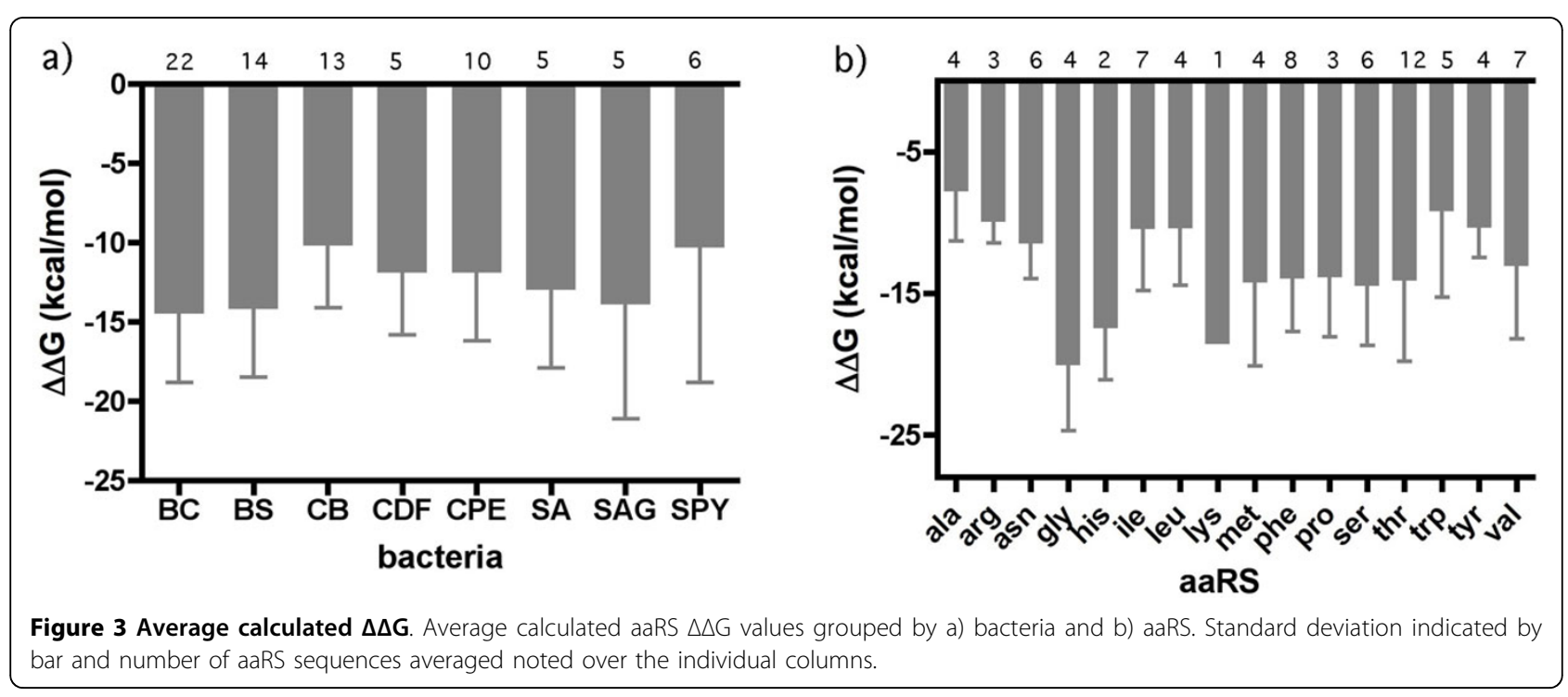


stability differences between these key riboswitch structural elements could potentially be targeted to effect ligand-specificity in future drug discovery efforts.

\section{Additional material}

\section{Additional file 1: Predicted $\Delta G$ values for aaRS $T$ box terminators} and antiterminators.

\section{Abbreviations}

(aaRS): Aminoacyl tRNA synthetase; (BC): Bacillus cereus; (BS): Bacillus subtilis; (CB): Clostridium botulinum; (CDF): Clostridium difficile; (CPE): Clostridium perfringens; Mu50 (SA): Staphylococcus aureus; (SAG): Streptococcus agalactiae and, Streptococcus pyogenes (SPY).

\section{Acknowledgements}

We wish to thank the National Institutes of Health (GM073188) for support of this work and the office of the VP for Research, Ohio University and the DAAD for support of FJ on an Internationale Studien- und

Ausbildungspartnerschaften (ISAP) grant.

This article has been published as part of BMC Bioinformatics Volume 13 Supplement 2, 2012: Proceedings from the Great Lakes Bioinformatics Conference 2011. The full contents of the supplement are available online at http://www.biomedcentral.com/bmcbioinformatics/supplements/13/s2

\section{Author details}

${ }^{1}$ Department of Chemistry, Leipzig University, Leipzig, Germany. ${ }^{2}$ Department of Chemistry \& Biochemistry, Ohio University, Athens, OH, USA.

\section{Authors' contributions}

FJ carried out the sequence folding and free energy predictions. JH conceived of the study, designed and coordinated the study and prepared the manuscript.

\section{Competing interests}

$\mathrm{JH}$ is a co-inventor on U.S. Patent number 7,005,441 for T box binding assays and ligands.

Published: 13 March 2012

\section{References}

1. Gutierrez-Preciado A, Henkin TM, Grundy FJ, Yanofsky C, Merino E: Biochemical features and functional implications of the RNA-based T box regulatory mechanism. Microbiol Mol Biol Rev 2009, 73:36-61.

2. Vitreschak AG, Mironov AA, Lyubetsky VA, Gelfand MS: Comparative genomic analysis of T-box regulatory systems in bacteria. RNA 2008, 14:717-735.

3. Green NJ, Grundy FJ, Henkin TM: The T box mechanism: tRNA as a regulatory molecule. FEBS Lett 2010, 584(2):318-324.

4. Grundy FJ, Moir TR, Haldeman MT, Henkin TM: Sequence requirements for terminators and antiterminators in the $\mathrm{T}$ box transcription antitermination system: disparity between conservation and functional requirements. Nucl Acids Res 2002, 30(7):1646-1655.

5. Grundy FJ, Yousef MR, Henkin TM: Monitoring uncharged tRNA during transcription of the Bacillus subtilis glyQS gene. J Mol Biol 2005, 346:73-81.

6. Thomas JR, Hergenrother PJ: Targeting RNA with Small Molecules. Chem Rev 2008, 108(4):1171-1224

7. Gerdeman MS, Henkin TM, Hines JV: In vitro structure-function studies of the Bacillus subtilis tyrS mRNA antiterminator: Evidence for factor independent tRNA acceptor stem binding specificity. Nucleic Acids Res 2002, 30(4):1065-1072.

8. Gerdeman MS, Henkin TM, Hines JV: Solution structure of the $B$. subtilis T box antiterminator RNA: Seven-nucleotide bulge characterized by stacking and flexibility. J Mol Biol 2003, 326:189-201.

9. Means JA, Simson CM, Zhou S, Rachford AA, Rack J, Hines JV: Fluorescence probing of $\mathrm{T}$ box antiterminator RNA: Insights into riboswitch discernment of the tRNA discriminator base. Biochem Biophys Res Commun 2009, 389:616-621.

10. Jack KD, Means $J A$, Hines $J V$ : Characterizing riboswitch function: Identification of $\mathrm{Mg}^{2+}$ binding site in T box antiterminator RNA. Biochem Biophys Res Commun 2008, 370:306-310.

11. Fauzi $H$, Agyeman $A$, Hines $\mathrm{JV}$ : $T$ box transcription antitermination riboswitch: Influence of nucleotide sequence and orientation on tRNA binding by the antiterminator element. Biochim Biophys Acta 2009, 1789:185-191.

12. Fauzi $H$, Jack $K D$, Hines $J V$ : In vitro selection to identify determinants in tRNA for Bacillus subtilis tyrS T box antiterminator mRNA binding. Nucleic Acids Res 2005, 33:2595-2602.

13. Means JA, Hines JV: Fluorescence resonance energy transfer studies of aminoglycoside binding to a T box antiterminator RNA. Bioorg Med Chem Lett 2005, 15:2169-2172.

14. Anupam R, Denapoli L, Muchenditsi AM, Hines JV: Identification of neomycin B binding site in T box antiterminator model RNA. Bioorg Med Chem 2008, 16:4466-4470.

15. Means JA, Katz SJ, Nayek A, Anupam R, Hines JV, Bergmeier SC: Structure activity studies of oxazolidinone analogs as RNA-binding agents. Bioorg Med Chem Lett 2006, 16(13):3600-3604.

16. Anupam R, Bergmeier SC, Green NJ, Grundy FJ, Henkin TM, Means JA, Nayek A, Hines JV: 4,5-Disubstituted Oxazolidinones: High affinity molecular effectors of RNA function. Bioorg Med Chem Lett 2008, 18:3541-3544.

17. Acquaah-Harrison G, Zhou S, Hines JV, Bergmeier SC: Library of 1,4disubstituted 1,2,3-triazole analogs of oxazolidinone RNA-binding agents. J Comb Chem 2010, 12:491-496.

18. Orac CM, Zhou S, Means JA, Boehme D, Bergmeier SC, Hines JV: Synthesis and stereospecificity of 4,5-disubstituted oxazolidinone ligands binding to T-box riboswitch RNA. J Med Chem 2011, DOl: 10.1021/jm2006904.

19. Maciagiewicz I, Zhou S, Bergmeier SC, Hines JV: Structure activity studies of RNA-binding oxazolidinone derivatives. Bioorg Med Chem Lett 2011 21:4524-4527.

20. Zhou S, Acquaah-Harrison G, Bergmeier SC, Hines JV: Anisotropy studies of tRNA - T box antiterminator RNA complex in the presence of 1,4disubstituted 1,2,3-triazoles. Bioorg Med Chem Lett 2011, DOI: 10.1016/j. bmcl.2011.1009.1095.

21. Markham NR, Zuker M: DINAMelt web server for nucleic acid melting prediction. Nucleic Acids Res 2005, 33(33 Web Server):W577-W581.

22. Zuker M, Stiegler P: Optimal computer folding of larger RNA sequences using thermodynamic and auxiliary information. Nucleic Acids Res 1981, 9(1):133-148

23. Zuker M, Mathew DH, Turner DH: Algorithms and thermodynamics for RNA secondary structure prediction: a practical guide. In RNA Biochemistry and Biotechnology. Kluwer Academic Publishers;Barciszewski J, Clark BFC 1999:11-43.

doi:10.1186/1471-2105-13-S2-S5

Cite this article as: Jentzsch and Hines: Interfacing medicinal chemistry with structural bioinformatics: implications for T box riboswitch RNA drug discovery. BMC Bioinformatics 2012 13(Suppl 2):S5.

\section{Submit your next manuscript to BioMed Central and take full advantage of:}

- Convenient online submission

- Thorough peer review

- No space constraints or color figure charges

- Immediate publication on acceptance

- Inclusion in PubMed, CAS, Scopus and Google Scholar

- Research which is freely available for redistribution 